Virginia Commonwealth University VCU Scholars Compass

Psychiatry Publications

Dept. of Psychiatry

2017

\title{
Somatic symptom disorder in dermatology
}

James L. Levenson

Virginia Commonwealth University

Aditi A. Sharma

Virginia Commonwealth University

Alex G. Ortega-Loayza

Oregon Health \& Science University School of Medicine

Follow this and additional works at: http://scholarscompass.vcu.edu/psych_pubs

Part of the Psychiatry and Psychology Commons

(C) 2017 Elsevier Inc. All rights reserved.

\section{Downloaded from}

http://scholarscompass.vcu.edu/psych_pubs/82

This Article is brought to you for free and open access by the Dept. of Psychiatry at VCU Scholars Compass. It has been accepted for inclusion in Psychiatry Publications by an authorized administrator of VCU Scholars Compass. For more information, please contact libcompass@vcu.edu. 


\title{
Somatic symptom disorder in dermatology
}

\section{James L. Levenson, MD ${ }^{a, *}$, Aditi A. Sharma, MD $^{b}$, Alex G. Ortega-Loayza, MD ${ }^{c}$}

\author{
a Department of Psychiatry, Virginia Commonwealth University School of Medicine, Richmond, Virginia \\ ${ }^{\mathrm{b}}$ Virginia Commonwealth University School of Medicine, Richmond, Virginia \\ ${ }^{\mathrm{c}}$ Department of Dermatology, Oregon Health \& Science University School of Medicine, Portland, Oregon
}

\begin{abstract}
Somatic symptom disorder (SSD) is defined by the prominence of somatic symptoms associated with abnormal thoughts, feelings, and behaviors related to the symptoms, resulting in significant distress and impairment. Individuals with these disorders are more commonly encountered in primary care and other medical settings, including dermatology practice, than in psychiatric and other mental health settings. What defines the thoughts, feelings, and behaviors as abnormal is that they are excessive, that is, out of proportion to other patients with similar somatic symptoms, and that they result in significant distress and impairment. SSD may occur with or without the presence of a diagnosable dermatologic disorder. When a dermatologic disorder is present, SSD should be considered when the patient is worrying too much about his or her skin, spending too much time and energy on it, and especially if the patient complains of many nondermatologic symptoms in addition. The differential diagnosis includes other psychiatric disorders, including depression, anxiety disorders, delusions of parasitosis, and body dysmorphic disorder.

This paper describes SSD and its applicability in dermatologic practice, with illustrative cases.

(C) 2017 Elsevier Inc. All rights reserved.
\end{abstract}

\section{Somatic symptom disorder}

The distinguishing characteristic of somatic symptom disorder (SSD) lies in the way the patient presents, interprets, and responds to the symptoms rather than to the somatic symptoms themselves. As a result, the diagnostic criteria for SSD include disproportionate emotional, cognitive, and behavioral manifestations, key to defining the disorders. ${ }^{1-4}$

The creation of SSD is due to the changes that occurred from DSM-IV to DSM-5. ${ }^{1,2}$ In DSM-IV, psychiatric conditions primarily presenting in medical settings with physical symptoms were grouped together as the "somatoform disorders," which included somatization disorder, hypochondriasis, pain disorders, and conversion disorder. The authors of DSM-

* Corresponding author. Tel.: +1 8048280763.

E-mail address: james.levenson@vcuhealth.org (J.L. Levenson).
5 recognized the overlap between the somatoform disorders and a lack of clarity about the boundaries of these DSM-IV diagnoses. DSM-5 sought to reduce the total number of disorders, as well as their subcategories, to make diagnosis more straightforward for nonpsychiatric physicians encountering patients with these symptoms. Patients who previously would have been found to have somatization disorder and most of those with hypochondriasis or pain disorders now have the new diagnosis of SSD.

The change is not just semantic but conceptual. Previous (DSM-III, DSM-IIIR, and DSM-IV) criteria emphasized the centrality of medically unexplained symptoms in diagnosing somatoform disorders. DSM-5 removed this requirement for several reasons. The reliability of determining that a somatic symptom is medically unexplained is limited, and grounding a diagnosis in the absence of an explanation is problematic and reinforces mind-body dualism. A mental disorder diagnosis should not be made solely, because a medical cause for 
Table 1 Diagnostic criteria for somatic symptom disorder

A. One or more somatic symptoms that are distressing or result in significant disruption of daily life

B. Excessive thoughts, feelings, or behaviors related to the somatic symptoms or associated health concerns as manifested by at least one of the following:

a. Disproportionate and persistent thoughts about the seriousness of one's symptoms

b. Persistently high level of anxiety about health or symptoms

c. Excessive time and energy devoted to these symptoms or health concerns

C. Although any one somatic symptom may not be continuously present, the state of being symptomatic is persistent (typically more than 6 months).

Specify if:

With predominant pain (previously pain disorder): This specifier is for individuals whose somatic symptoms predominantly involve pain. Specify if:

Persistent: A persistent course is characterized by severe symptoms, marked impairment, and long duration (more than 6 months).

Specify current severity:

Mild: Only one of the symptoms specified in criterion B is fulfilled.

Moderate: Two or more of the symptoms specified in criterion B are fulfilled.

Severe: Two or more of the symptoms specified in criterion B are fulfilled, plus there are multiple somatic complaints (or one very severe somatic symptom).

Reproduced with permission from the Diagnostic and Statistical Manual of Mental Disorders, 5th ed. (Copyright @ 2013), American Psychiatric Association. All Rights Reserved.

physical signs and symptoms cannot be shown. In addition, the presence of a medical diagnosis does not exclude the possibility of a comorbid mental disorder. A psychiatric diagnosis should be based primarily on the presence of abnormal psychologic symptoms and signs.

The new (DSM-5) ${ }^{1}$ classification defines the major diagnosis of SSD on the basis of positive symptoms, namely, distressing somatic symptoms plus abnormal thoughts, feelings, and behaviors in response to these symptoms, rather than an absence of a medical explanation for the somatic symptoms. What defines the thoughts, feelings, and behaviors as abnormal is that they are excessive, that is, out of proportion to other patients with similar somatic symptoms, and that they result in significant distress and impairment.

Some mental disorders, including major depressive disorder or panic disorder, may initially manifest with primarily somatic symptoms. Such diagnoses may account for the somatic symptoms, or they may be comorbid with one of the somatic symptom and related disorders. The somatic component adds severity and complexity to depressive and anxiety disorders and results in higher severity, functional impairment, and even refractoriness to traditional treatments. The criteria for SSD are shown in Table 1. The prevalence of SSD is unknown, but in the general adult population it may be around $5-7 \%$. Women tend to report more somatic symptoms.

Several factors contribute to somatic symptom and related disorders, including genetic and biologic vulnerability, early traumatic experiences, and learning, as well as cultural/social norms that devalue and stigmatize psychologic suffering compared with physical suffering. Variations in symptom presentation are often due to the interaction of many factors within cultural contexts that influence how a person may identify or classify sensation, interpret illness, and seek medical help. SSD occurs across the age spectrum. ${ }^{5}$

The core features of SSD are multiple somatic symptoms (or one severe symptom) that are distressing and result in significant impairment: Worrying too much about the somatic symptoms, plus spending too much time and energy on them. In dermatology practice, the somatic symptoms may be entirely focused on the skin, with pruritus or pain being most common, or the cutaneous symptoms may accompany other symptoms, such as headache, back pain, fatigue, gastrointestinal symptoms, chest pain, shortness of breath, and paresthesias.

In addition to multiple symptoms, SSD patients often present vague and inconsistent histories expressing dissatisfaction with previous care received. They are often viewed as "difficult patients." The symptoms and related concerns often dominate the patient's life, and psychosocial functioning declines. Many patients engage in excessive self-monitoring to reassure themselves, for example, checking their skin frequently or repeatedly measuring blood pressure and pulse. Patients with SSD often seek remedies through complementary/alternative practitioners, and consume vitamins, over-the-counter remedies, and fad diets. Many patients devote excessive amounts of time on the Internet reading about medical diseases and treatments.

SSD can occur with or without a general medical illness that can "explain" their somatic symptoms. When such an illness is present (eg, psoriasis), SSD can be diagnosed when the patient's cognitive-emotional-behavioral response to the skin disease is clearly excessive compared with most other patients with that same skin disorder of similar severity. Patients with 
SSD often have significant depression and/or anxiety and may qualify for an additional psychiatric diagnosis. Patients with more severe SSD also frequently have personality disorders, in addition to depression and anxiety, making their management more difficult. Patients with SSD vary in their degree of insight. Some recognize that they have overreacted to their physical symptoms, whereas others do not see that their reactions are excessive; however, their beliefs about their symptoms are not delusional; that is, their somatic symptoms and worries are plausible and not bizarre.

The excessive focus on their symptoms and the high level of health anxiety often result in high health care utilization. Multiple physical examinations and tests that are explained as normal typically do not allay the patient's concern. Doctor-shopping, duplicated progressively more invasive diagnostic tests (eg, biopsy of skin, lymph node, and nerve), and higher-risk treatments may result, which can reinforce the sick role and increase the risk of iatrogenic complications. Despite extensive medical attention, patients with SSD often are disappointed and frustrated with the care they have received. Physicians become frustrated, because their efforts to help and reassure the patient are ineffective.

The diagnosis of SSD requires taking a full history, including a review of systems (not just the presenting dermatologic symptom), performing a physical examination, and reviewing prior medical care. Many will have seen several prior physicians and have been dissatisfied. The history may reveal a pattern of multiple physical symptoms, excessive health care utilization, doctor-shopping, and greater psychosocial impairment than expected. Even when the physical complaint seems grossly exaggerated, a medical etiology should be considered so that patients feel that they are being taken seriously, and because SSD can be a maladaptive response to a significant medical illness. Establishing trust and avoiding the patients feeling disrespected or humiliated are essential. Telling patients that there is nothing wrong with them or that they are making too big a deal out of their symptoms is not helpful and risks further antagonizing patients.

In taking a history of the present illness, the dermatologist should note how the physical symptoms are related to the patient's life circumstances and emotions, particularly if any stressful events have occurred. What exacerbates or alleviates the symptoms? What do patients think is wrong and how seriously ill do they think they are? How much time, energy, worry, and thought do they devote to the somatic symptoms? The physical examination should help to satisfy the physician that the patient does not have a medical disease, or if there is one, provide a perspective on its severity. Patients are more likely to believe that their complaints are taken seriously when a physical examination is performed. Decisions about diagnostic tests, especially invasive ones, should be based on objective signs rather than on the volume of the symptom complaint expressed by the patient. Physicians often bear responsibility for pursuing excessive diagnostic testing, even when there are clear indications that the etiology is psychiatric. ${ }^{6,7}$ Diagnostic tests are often ordered to provide reassurance, even when the physician has concluded that there is no significant risk of serious disease. ${ }^{8}$ Unfortunately, such testing does not reassure patients. ${ }^{9}$

How should the diagnosis of SSD be discussed with patients? Regardless of suspected etiology, physicians should convey to patients that they regard the symptoms as real (eg, they are not "all in your head"). When no medical disorder has been identified, physicians should avoid debating whether the symptoms are due to a medical or psychiatric cause. Directly challenging patients' beliefs about their symptoms may undermine the doctor-patient relationship. When there is an identified medical disorder about which patients are disproportionately concerned, it can be counterproductive to tell them that their complaints have been blown out of proportion, as this risks their feeling discounted and disrespected. Instead, one should start with empathic reflection that recognizes the burden the somatic symptoms have been on the patient. This may be followed by an explanation of how sensitivity to pain, itching, or other physical symptoms is affected by stress and coping style.

The differential diagnosis of SSD includes both medical and psychiatric disorders. ${ }^{1}$ If the somatic symptoms are consistent with another mental disorder and the diagnostic criteria for that disorder are satisfied, the mental disorder should be considered as an alternative diagnosis. Patients can have both SSD and another psychiatric disorder simultaneously.

1. Panic disorder: In panic disorder, the somatic symptoms and anxiety regarding health occur in acute intermittent episodes, as opposed to SSD, where the anxiety and somatic symptoms remain persistent.

2. Generalized anxiety disorder: In generalized anxiety disorder there is worry about multiple events, situations, or activities, which may involve their health. The focus is less likely to remain on somatic symptoms or fear of illness as it is in SSD.

3. Depressive disorders: Depressive disorders may present with somatic symptoms; however, the distinction lies in the presence of core depressive symptoms of dysphoria and anhedonia.

4. Illness anxiety disorder: Illness anxiety disorder involves extensive worry about health without symptoms or with minimal somatic symptoms.

5. Conversion disorder: Conversion disorder includes the loss of function, whereas in SSD the emphasis remains on the distress that the particular symptom is causing. Criterion B helps to distinguish these two disorders.

6. Delusional disorder: In SSD, the individual's beliefs that the somatic symptoms might reflect serious underlying physical illness are not held with delusional intensity. In delusional disorder, somatic subtype, the somatic symptom beliefs and behavior are stronger than those found in SSD.

7. Body dysmorphic disorder: In body dysmorphic disorder, the individual is excessively preoccupied with a perceived defect in physical features. In SSD, the 
concern is with underlying illness compared with physical appearance.

8. Obsessive-compulsive disorder: In SSD, there is less severity in the recurrence of ideas about the somatic symptoms and there are no associated repetitive behaviors to reduce anxiety, as seen in obsessive-compulsive disorder.

\section{Case examples}

\section{A patient with SSD and minor dermatologic disease}

\section{Case 1}

A 51-year-old woman with diabetes mellitus, peripheral neuropathy, hyperlipidemia, hypertension, and low back pain presented for evaluation of "warts" on her hands and feet. The "warts" began in 2008, which the patient attributed to working as a nurse. She described the plantar warts as painful and worsened by walking or even standing. During the interview, the patient pointed to numerous places on her palms, fingers, and dorsal distal interphalangeal (DIP) joints. When she applied aloe vera, they "popped out." They could move from one place to another, and she heard them popping when she rubbed her skin. She washed her hands several times daily after going to the bathroom. The patient had seen numerous doctors who, she reported, thought she was "crazy" and prescribed her lorazepam. In 2014, a dermatologist performed cryotherapy, providing some relief. She expressed interest in repeat cryotherapy.

\section{Physical examination}

On initial examination, her hands showed a few small 1-mm vesicles, despite the patient pointing to various locations of "warts" that appeared to be clinically normal skin. Two vesicles were noted on the left palmar thenar eminence, and one was noted on the left middle digit (palmar aspect). The palmar aspect of both hands showed mild diffuse hyperkeratinization. In addition, bony overgrowth of DIP joints and diffuse verrucous hyperkeratinization was appreciated. The patient had a hyperpigmented macule on her thumb, which the patient considered a collection of warts. On her feet, diffuse hyperkeratosis of pressure areas on the plantar feet was present. She was alert and oriented and maintained a cooperative, appropriate mood and affect.

\section{Discussion}

This represents SSD with a nonspecific dermatitis as the cause of her symptoms. She misperceives a normal variation in her skin as evidence of significant pathology. She presents with significant pain and is very concerned about recurrent "warts." She does have dyshidrosis with some mild postinflammatory hyperpigmentation, but she does not have evidence of verrucae. She has consulted many physicians and pushes for multiple interventions.
The best approach would be to redirect the patient by thoroughly explaining the most likely diagnosis: Dyshidrosis.

\section{Patients with significant dermatologic disease whose thoughts, emotions, and/or behavior are out of proportion to their dermatologic disease}

\section{Case 2}

A 45-year-old woman with anxiety presented to the dermatology clinic with a generalized eruption present for 4 months and induced by insect bites. She had seen another physician 1 week after the onset, who confirmed an insect bite reaction. Topical steroids were moderately helpful; however, she continued to feel tingling with "pins and needles" sensations. In addition, she noticed linear skin eruptions on her face and scalp, making her extremely concerned that these might represent something more serious or even life threatening that now interfered with her work. She feels that previous medical evaluation and treatment have not been adequate and is very worried that her symptoms represent something more serious than bug bites.

\section{Physical examination}

On initial examination, there were scattered erythematous papules with linear crusted papules, some confluent on the right leg. She had 5/5 strength in the upper and lower extremities. Light touch, pinprick, position sense, and vibration sense were intact in fingers and toes. She was alert and oriented and seemed cooperative, with appropriate mood and affect.

\section{Discussion}

This patient anxiously presented to clinic for nonspecific dermatitis concerned that something more serious was affecting her. If the objective findings on examination had been more concerning, then it would have been appropriate to do further medical evaluation; however, in this case there did not appear to be an indication for further testing. The patient showed thoughts, feelings, and behaviors that were out of proportion to other patients with similar somatic symptoms of a diagnosable dermatologic disease, in this case insect bites, and it resulted in significant distress and impairment, consistent with SSD.

\section{Case 3}

A 48-year-old woman with fibromyalgia and questionable attention-deficit/hyperactivity disorder presented with a 3-year history of upper torso flushing with diaphoresis of the upper half of her body, and pruritus with crawling sensations on her forehead. She previously underwent an extensive evaluation by endocrinology for carcinoid syndrome, pheochromocytoma, and hyperthyroidism but ultimately attributed her symptoms to methylphenidate taken for attention-deficit/hyperactivity disorder. The symptoms persisted after discontinuation of the drug. 
Concurrently, a previous dermatologist had found her to have rosacea. She was looking for a second opinion because she was dissatisfied with both diagnoses. From her reading on the Internet she was very concerned about having cancer, and she complained that the previous physicians had not listened to her. She insisted on further laboratory tests and imaging.

\section{Physical examination}

On physical examination, there were mild erythematous patches on the forehead, cheeks, and chin. No other skin changes were noted. She was alert and oriented, anxious, and exhibited appropriate mood.

\section{Discussion}

It is common in patients with SSD that physicians devote excessive attention to ruling out rare disorders as explanation for the patient's difficult-to-explain symptoms, in this case carcinoid syndrome and pheochromocytoma. This led to the patient continuing to worry that she had an occult disorder even after extensive evaluation had found no evidence.

The distinguishing characteristic of SSD lies in the way the patient presents, interprets, and responds to the symptoms rather than the somatic symptoms themselves. As such, patients will exhibit disproportionate emotional, cognitive, and behavioral manifestations, key to defining the disorder. This patient shows disproportionate behavioral and cognitive manifestations such as seeking multiple opinions, requesting additional imaging, and doing extensive research on her own to explain her symptoms.

Patients' symptoms should never be discounted solely due to atypical presentation or previous psychiatric history. In this case, the patient started out with a dermatologic symptom of probable drug-induced flushing; however, she continued to complain of symptoms even after the medication was stopped. She continued to worry despite the negative medical workup and her physicians' reassurance, thus pointing to SSD. The role of the dermatologist was to explain rosacea, and that flushing can be benign.

\section{Examples that are not SSD}

\section{Case 4}

A 50-year-old man with a history of hemochromatosis presented with chronic cutaneous ulcers on his neck and face, with a fluctuating course alternating between healing and breaking open. He had seen several providers for this and has had a negative skin biopsy. He has taken several courses of oral and IV antibiotics, and chronically used topical bacitracin. He is concerned that there are parasites in the wound, and admits to frequently scratching and removing what he believes are insect eggs. He has brought samples along with several pictures of the "bugs." Despite multiple providers' reassurance, he continues to insist that he is infested.

\section{Physical examination}

On physical examination, there was a very large ulcer with surrounding erythema on his right neck, and a large irregularly shaped hypopigmented scar on his left neck. He also had a superficial ulcer on his left cheek, along with a few shallow ulcers on his left leg. He was alert and oriented, seemed cooperative, and exhibited appropriate mood and affect.

\section{Discussion}

This is a case of a 50-year-old man with a classic history and findings consistent with delusions of parasitosis as the likely diagnosis. When a different psychiatric diagnosis accounts for a patient's symptoms, it should be used rather than SSD. In this case the patient has delusional beliefs about infestation and in DSM-5 would be diagnosed as having a delusional disorder, somatic type.

How to manage delusions of parasitosis is outside the scope of this paper (see a recent review for practical suggestions ${ }^{10}$ ), but in such patients it is always important to be on the lookout for superimposed staphylococcal or other secondary infections, or underlying systemic illnesses (eg, hepatic or renal insufficiency, stimulant abuse) that can cause secondary delusions of parasitosis.

\section{Conclusions}

When a patient is encountered in dermatology practice with prominent cutaneous symptoms causing the patient significant distress and/or impairment, either in the absence of objective signs of disease, or grossly disproportionate to a diagnosed dermatologic condition, the diagnosis of SSD should be considered. The key features of SSD are the abnormal and excessive thoughts (eg, constant worry that one has cancer), feelings (eg, overwhelming anxiety), and behaviors (eg, constant checking of one's skin) related to the symptoms. The presence of many nondermatologic symptoms in addition to the cutaneous ones is further suggestive of SSD. The differential diagnosis includes other psychiatric disorders frequently seen in dermatology, including depression, anxiety disorders, obsessive-compulsive disorder, delusions of parasitosis, and body dysmorphic disorder. After taking a history, careful examination and empathic explanation to the patient can avoid unnecessary, costly, and potentially harmful testing and biopsies.

\section{Disclosures}

The authors have no financial relationships relevant to the paper to disclose. 


\section{References}

1. American Psychiatric Association. Diagnostic and Statistical Manual of Mental Disorders. 5th ed. Arlington, VA: American Psychiatric Association; 2013.

2. Dimsdale JE, Levenson J. What's next for somatic symptom disorder? Am J Psychiatry. 2013;170:1393-1395.

3. Barsky AJ. Assessing the new DSM-5 diagnosis of somatic symptom disorder. Psychosom Med. 2016;78:2-4.

4. Rief W, Martin A. How to use the new DSM-5 somatic symptom disorder diagnosis in research and practice: A critical evaluation and a proposal for modifications. Annu Rev Clin Psychol. 2014;10: 339-367.

5. van Geelen SM, Rydelius PA, Hagquist C. Somatic symptoms and psychological concerns in a general adolescent population: Exploring the rel- evance of DSM-5 somatic symptom disorder. J Psychosom Res. 2015;79: 251-258.

6. Bensing JM, Verhaak PF. Somatisation: A joint responsibility of doctor and patient. Lancet. 2006;367:452-454.

7. Ring A, Dowrick CF, Humphris GM, et al. The somatising effect of clinical consultation: What patients and doctors say and do not say when patients present medically unexplained physical symptoms. Soc Sci Med. 2005;61:1505-1515.

8. Kroenke K. Diagnostic testing and the illusory reassurance of normal results: Comment on "Reassurance after diagnostic testing with a low pretest probability of serious disease". JAMA Intern Med. 2013;173:416-417.

9. Rolfe A, Burton C. Reassurance after diagnostic testing with a low pretest probability of serious disease: Systematic review and meta-analysis. JAMA Intern Med. 2013;173:407-416.

10. Patel V, Koo JY. Delusions of parasitosis; suggested dialogue between dermatologist and patient. J Dermatol Treat. 2015;26:456-460. 